\title{
Characterization and treatment monitoring of inflammatory arthritis by photoacoustic imaging: a study on adjuvant-induced arthritis rat model
}

\author{
Justin R. Rajian, ${ }^{1}$ Xia Shao, ${ }^{1}$ David L. Chamberland, ${ }^{2}$ and Xueding Wang ${ }^{1, *}$ \\ ${ }^{I}$ Department of Radiology, University of Michigan School of Medicine, Ann Arbor, MI 48109, USA \\ ${ }^{2}$ Division of Rheumatology, University of Michigan School of Medicine, Ann Arbor, MI 48109, USA \\ *xdwang@umich.edu
}

\begin{abstract}
Neovascularity also known as angiogenesis is an early feature of inflammatory arthritis disease. Therefore, identifying the development of neovascularity is one way to potentially detect and characterize arthritis. Laser-based photoacoustic imaging (PAI) is an emerging biomedical imaging modality which may aid in the detection of both early and continued development of neovascularity. In this work, we investigated the feasibility of PAI to measure angiogenesis, for the purpose of evaluating and monitoring inflammatory arthritis and responses to treatment. The imaging results on an arthritis rat model demonstrate that 1) there is noticeable enhancement in image intensities in the arthritic ankle joints when compared to the normal joints, and 2) there is noticeable decrease in image intensities in the arthritic ankle joints after treatment when compared to the untreated arthritic joints. In order to validate the findings from PAI, we performed positron emission tomography (PET) and histology on the same joints. The diameters of the ankle joints, as a clinical score of the arthritis, were also measured at each time point.
\end{abstract}

(C)2013 Optical Society of America

OCIS codes: (170.5120) Photoacoustic imaging; (110.7170) Ultrasound.

\section{References and links}

1. Centers for Disease Control and Prevention (CDC), "Racial/ethnic differences in the prevalence and impact of doctor-diagnosed arthritis - United States, 2002," MMWR Morb. Mortal. Wkly. Rep. 54(5), 119-123 (2005).

2. O. Fitzgerald and B. Bresnihan, "Synovial vascularity is increased in rheumatoid arthritis: comment on the article by Stevens et al," Arthritis Rheum. 35(12), 1540-1541 (1992).

3. A. E. Koch, "Review: angiogenesis: implications for rheumatoid arthritis," Arthritis Rheum. 41(6), 951-962 (1998).

4. A. J. Hayes, "Angioneogenesis in rheumatoid arthritis," Lancet 354(9176), 423-424 (1999).

5. E. M. Paleolog and J. M. Miotla, "Angiogenesis in arthritis: role in disease pathogenesis and as a potential therapeutic target," Angiogenesis 2(4), 295-307 (1998).

6. D. A. Walsh, "Angiogenesis and arthritis," Rheumatology (Oxford) 38(2), 103-112 (1999).

7. A. E. Koch, "Angiogenesis as a target in rheumatoid arthritis," Ann. Rheum. Dis. 62(90002 Suppl 2), ii60-ii67 (2003).

8. P. H. Wooley, “Animal models of rheumatoid arthritis," Curr. Opin. Rheumatol. 3(3), 407-420 (1991).

9. M. M. Halloran, J. M. Woods, R. M. Strieter, Z. Szekanecz, M. V. Volin, S. Hosaka, G. K. Haines 3rd, S. L. Kunkel, M. D. Burdick, A. Walz, and A. E. Koch, "The role of an epithelial neutrophil-activating peptide-78-like protein in rat adjuvant-induced arthritis," J. Immunol. 162(12), 7492-7500 (1999).

10. S. Shahrara, A. E. I. Proudfoot, J. M. Woods, J. H. Ruth, M. A. Amin, C. C. Park, C. S. Haas, R. M. Pope, G. K. Haines, Y. Y. Zha, and A. E. Koch, "Amelioration of rat adjuvant-induced arthritis by Met-RANTES," Arthritis Rheum. 52(6), 1907-1919 (2005).

11. M. M. Halloran, Z. Szekanecz, N. Barquin, G. K. Haines, and A. E. Koch, "Cellular adhesion molecules in rat adjuvant arthritis," Arthritis Rheum. 39(5), 810-819 (1996).

12. J. D. Taurog, D. C. Argentieri, and R. A. McReynolds, "Adjuvant arthritis," Methods Enzymol. 162, 339-355 (1988). 
13. D. A. Barnes, J. Tse, M. Kaufhold, M. Owen, J. Hesselgesser, R. Strieter, R. Horuk, and H. D. Perez, "Polyclonal antibody directed against human RANTES ameliorates disease in the Lewis rat adjuvant-induced arthritis model,” J. Clin. Invest. 101(12), 2910-2919 (1998).

14. X. Wang, J. B. Fowlkes, J. M. Cannata, C. Hu, and P. L. Carson, "Photoacoustic imaging with a commercial ultrasound system and a custom probe," Ultrasound Med. Biol. 37(3), 484-492 (2011).

15. M. K. Chen and T. R. Guilarte, "Translocator protein $18 \mathrm{kDa}$ (TSPO): molecular sensor of brain injury and repair," Pharmacol. Ther. 118(1), 1-17 (2008).

16. V. Papadopoulos and L. Lecanu, "Translocator protein $(18 \mathrm{kDa})$ TSPO: an emerging therapeutic target in neurotrauma,” Exp. Neurol. 219(1), 53-57 (2009)

\section{Introduction}

Affecting approximately 46 million Americans, arthritis is the leading cause of disability, and is associated with substantial activity limitation, work disability, reduced quality of life, and high health-care costs [1]. Medical Expenditure Panel Survey data indicates that during 2003 the total cost of arthritis and other rheumatic conditions in the U.S. was approximately $\$ 128$ billion, equivalent to $1.2 \%$ of the gross domestic product and second only to cardiovascular diseases. Although arthritis is serious, potentially crippling, and commonly disabling, objective characterization and optimized therapies of these disorders are hindered drastically by lack of cost efficient and powerful joint imaging technologies. The development of optimized therapies to prevent or delay disease progression and the prediction of those who will or will not respond to treatments are common goals for research and industry. However, many new pharmaceutical and non-pharmaceutical therapies have not been brought to the clinic, in large part because of the inability to accurately measure disease progression and response to these therapies.

Finding new biomarkers of arthritic diseases and developing novel medical imaging technologies that can assess these biomarkers in living organs become increasingly important for accurate diagnosis and sensitive monitoring of therapies. In the early stage of inflammatory arthritis, angiogenesis and increased vascularity is noted in and around the synovium within the affected joints [2-7]. Blood contains a variety of chromophores such as oxygenated hemoglobin, deoxygenated hemoglobin, both are strongly absorptive in the visible to near-infrared (NIR) spectral region. Optical imaging modalities such as PAI are highly desirable for inflammatory arthritis diagnosis, because optical contrast is intrinsically sensitive in mapping the dynamic distribution of these chromophores. For the first time, important physiological biomarkers of synovitis such as neoangiogenesis, hyperemia, and hypoxia in regional synovial tissues may be quantitatively analyzed by using PAI. In comparison with conventional diffuse optical imaging, PAI is capable of obtaining richer anatomical details due to its higher spatial resolution. Imaging of the above mentioned biomarkers with high spatial resolution especially benefits the study of small joint structures of human hands and feet which are among the earliest to be affected by some types of inflammatory arthritis including rheumatoid arthritis.

In this study, we have examined the capability of PAI in evaluating angiogenesis in articular tissues in vivo for the purpose of diagnosing and monitoring the treatment of inflammatory arthritis. This study was conducted on a well-established adjuvant-induced arthritis (AIA) rat model which is a rodent model similar clinically and pathologically to human rheumatoid arthritis [8-10]. To quantify the photoacoustic signal enhancement in the arthritic joint as a result of angiogenesis, the ankle joints of the rat were imaged at $532 \mathrm{~nm}$. PAI of a joint was achieved by using a commercial ultrasound (US) unit without affecting its original imaging functions. This arrangement enables the same object to be scanned in both US and PAI modes using the same receiving probe at the same viewing angle, which facilitates very convenient off-line image co-registration. US imaging, which can visualize the morphological joint features well, can be used to guide PAI procedure and help to analyze the functional findings from PAI. In order to validate the outcomes from the PAI technique, we also imaged the same samples with PET which, although involving ionizing radiation, has proved excellent sensitivity for inflammation and, therefore, was employed as a gold standard. The histological examination and the recording of clinical score of the imaged joints were also conducted, to verify angiogenesis and confirm arthritis in the joints. 


\section{Materials and methods}

\subsection{Animal model}

Female Lewis rats with body weights of $150 \mathrm{~g}$ were obtained from Charles River Laboratory. To induce AIA, each rat, on day 0, was injected subcutaneously into the base of the tail with lyophilized mycobacterium butyricum (DIFCO Labs, New York, NY) suspended in mineral oil [11]. $15 \mathrm{mg}$ powdered mycobacterium butyricum were added in $2 \mathrm{ml}$ of mineral oil (Paraffin oil, Fisher Scientific) and grinded well in a mortar and pestle for about thirty minutes. For each rat, $0.4 \mathrm{ml}$ of this suspension was injected into the base of the rat tail under aseptic condition. 20 days after adjuvant injection, a steep increase in clinical score was observed. More than $90 \%$ of the rats developed arthritis, showing joint histological changes including leukocyte invasion preceding joint swelling [12,13]. Before imaging the ankle joint, hair on the rat legs in the region of interest were completely removed by applying hair removal lotion to avoid light and ultrasound blockage. Before the adjuvant injection and each imaging experiment, a dose of $87 \mathrm{mg} / \mathrm{kg}$ Ketamine plus Xylasine $13 \mathrm{mg} / \mathrm{kg}$ was administered intramuscularly to anesthetize the rat. To explore the feasibility of PAI in diagnosis of inflammatory arthritis, a total of 12 arthritic ankle joints from the AIA rats were imaged on day 20 after adjuvant injection and compared with the 12 normal joints from the control group.

\subsection{Treatment of arthritis}

To demonstrate the capability of PAI in evaluating the treatment efficacy, AIA rats in the treatment group were treated with etanercept (Enbrel®), an anti-tumor necrosis factor medication used for clinical management of rheumatoid arthritis, ankylosing spondylitis and psoriatic arthritis. Starting from day 20 after adjuvant injection, each AIA rat was treated four times with a constant interval of four days (i.e. treated on day 20, 24, 28, 32 after adjuvant injection, respectively). For each treatment, etanercept was administrated intramuscularly with a dose of $0.5 \mathrm{ml} / \mathrm{kg}$. On day 35, the ankle joints from the treatment group were imaged to compare with the results from the control group which was formed by AIA rats that were also on day 35 after adjuvant injection and had not been treated with any medication. University of Michigan animal care procedures and protocol were followed in handling the rats for all the experiments.

\subsection{PAI and US imaging}

Schematic of the experimental setup for PAI and US dual-modality imaging of a rat joint is given in Fig. 1. The light beam from the Nd:YAG laser (Continuum, Santa Clara, CA, USA), after being split into two by a 50/50 beam splitter, was delivered to the imaged joint. Illumination from both sides of the joint helps to achieve a homogenous light fluence in the imaging plane and also keep the energy density on the skin well below the ANSI safety limit. During image acquisition, the rat leg immersed in water was fixed on a platform with an angle of inclination of $30^{\circ}$. The laser generated photoacoustic signals were acquired by a commercial US unit (z.one, Zonare Medical Systems, Inc., Mountain View, CA, USA) using a L10-5 linear probe. The probe, with a total of 128 elements and a detection bandwidth from 5 to $10 \mathrm{MHz}$, was placed about $2 \mathrm{~cm}$ above the rat leg with intervening water and aligned parallel to the leg. In order to ensure that all the images were obtained in the similar condition, the sample platform was tightly fixed and marked so that the rat legs can be positioned almost the same every time. Further, the positions of the sample platform, the laser beam and the transducer were fine-tuned, which also helped to achieve the similar condition for each scan. Moreover, we have taken into account the laser fluctuation by dividing the photoacoustic signal intensities by the laser energy measured during each scan.

Before PAI, gray-scale US imaging of the target joint was conducted first to find the best view in visualizing articular tissues. Then, without moving the sample and the probe, photoacoustic images were acquired from the same joint. To achieve a better signal-to-noise ratio, each PAI image was averaged over 100 frames. Since the US unit comes with 64 
independent channels, in order to acquire the data from all the 128 transducer elements, the US needs to run with the first and the second groups of 64 transducer elements respectively. With the current system, the time period for one 2D photoacoustic image is about 1 minute which is acceptable for in vivo imaging. By scanning the probe elevationally, 3D imaging is also technically possible which, however, needs a long acquisition time. Considering possible artifacts due to animal motions and health deterioration of animals as a result of the long signal acquisition, 3D imaging was not conducted in this study. The detailed technical aspects of this imaging system have been introduced in our previous publication [14]. In this study, the pulsed laser with pulse repetition rate of $10 \mathrm{~Hz}$ and pulse duration of $5.5 \mathrm{~ns}$ working at the wavelength of $532 \mathrm{~nm}$ was used. Since both oxygenated and deoxygenated hemoglobin absorb light strongly at $532 \mathrm{~nm}$, this wavelength is a good choice for blood-contrast imaging and the measurement of angiogenesis. Although the imaging depth in the visible spectrum is limited due to the strong attenuation of light in biological tissues, this depth is sufficient for the study of the small peripheral joints of rats.

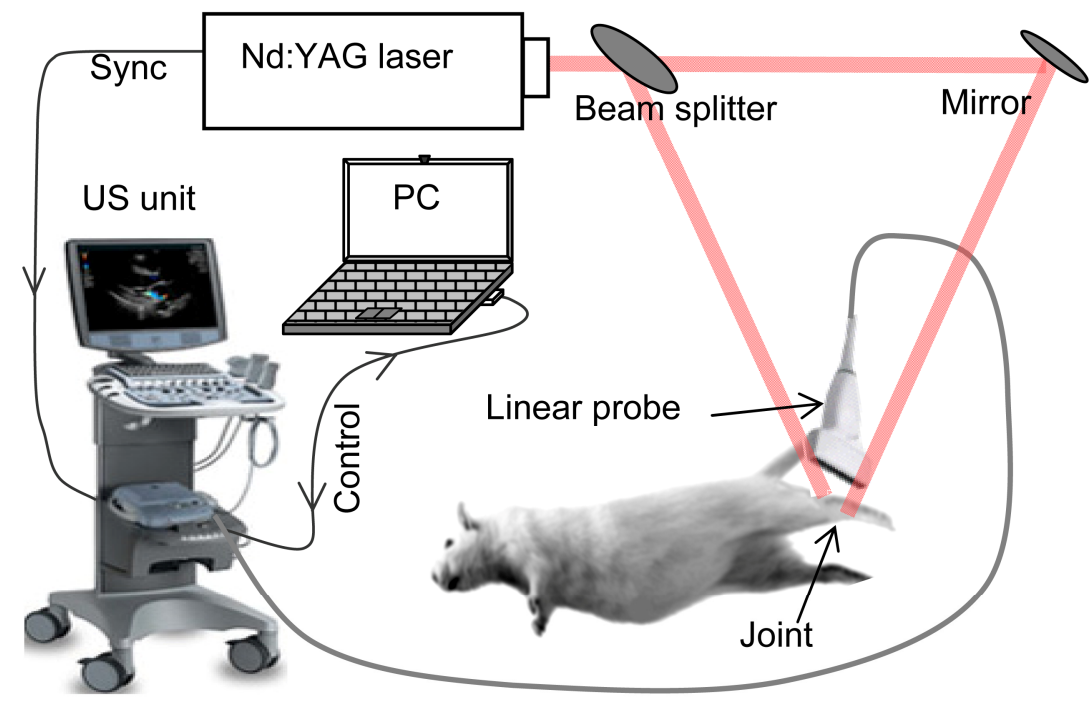

Fig. 1. Schematic of the experimental setup for PAI and US dual-modality imaging of a rat ankle joint.

\subsection{MicroPET}

Recently, the $18 \mathrm{kDa}$ translocator protein (TSPO), earlier called peripheral benzodiazepine receptor (PBR), has been investigated actively as a marker of reactive gliosis and inflammation $[15,16]$. It has been demonstrated that radiotracers binding to translocator protein (TSPO) can be used for in vivo imaging of rheumatoid synovitis. As an example, the study on rheumatoid arthritis patients concluded that ${ }^{11} \mathrm{C}$-(R)-PK11195 PET would be useful for both early detection of synovitis and monitoring of disease activity during treatment. Here, we used ${ }^{11}$ C-PBR28, a second generation PET radiotracer for imaging the TSPO receptors with improved bio-distribution, in the PET imaging of both normal and AIA rats with and without being treated with etanercept. With an excellent sensitivity to inflammation, the images from PET were employed as a gold standard to validate the findings from PAI.

Animals were anesthetized using an isoflurane anesthesia machine and placed prone on a microPET scanner (Rodent R4 microPET, Concorde Microsystem Inc., Knoxville, TN). After completion of the attenuation scan, animals were injected intravenously in the tail vein with a bolus of 11C-PBR28 ( 1 $\mathrm{mCi}$ in $0.2 \mathrm{ml}$ for each rat) and then a dynamic PET was performed for 60 minutes $(10 \times 30 \mathrm{sec}, 3 \times 1 \mathrm{~min}, 1 \times 2 \mathrm{~min}, 4 \times 5 \mathrm{~min}, 3 \times 10 \mathrm{~min})$. The field of view 
included abdomen, pelvis, proximal lower extremities and legs. Emission data were corrected for attenuation and scatter and reconstructed using the $3 \mathrm{D}$ maximum a priori method (3D MAP algorithm), yielding a reconstructed image resolution of approximately $1.4 \mathrm{~mm}$.

\subsection{Histology and clinic score}

To confirm arthritis in the joints and verify angiogenesis, standard histology examination of imaged ankle joints was conducted after the imaging experiment. Whole legs were harvested and fixed in $10 \%$ neutral buffered formalin for 48 hours. Tissues were decalcified for 5 days in a formic acid-based decalcifying solution (Immunocal, Decal Chemical Co., Tallman, NY) followed by rinsing and processing to paraffin by standard histological methods. Hematoxylin-and-eosin stained slides were evaluated by a board-certified veterinary pathologist on an Olympus BX-45 light microscope. Photos were taken using an Olympus DP-72 microscope-mounted 12.5 megapixel digital camera.

To confirm arthritis in the affected joints, clinical evaluation of the degree of arthritis was also conducted. The sizes of the ankle joints, as a clinical score of the arthritis, were measured at each time point. In order to physically quantify the severity of arthritis, the diameter of the rat ankle joint was measured by using a caliper. Two measurements were made on the vertical and horizontal directions respectively, and then an average was taken as the diameter of the ankle joint.

\section{Results}

Typical PAI and US images of an arthritic ankle joint are given in Fig. 2. These images were taken on day 20 after adjuvant injection. Despite that PAI and US are based on different contrasts, there is some resemblance between the two images. In the US image, we can recognize the target joint clearly, which enables the selection of the region of interest (ROI) in both US and PAI images, as indicated by the dotted squares. In PAI image, the intensity demonstrates the optical energy deposition in the tissue surrounding the joint. After compensation of the light fluence, this image enables the assessment of the hemoglobin related optical absorption in joint tissues, which can help evaluate the angiogenesis as a result of the inflammatory arthritis. Since the PAI and US images were acquired using the same system, they are co-registered naturally. As shown in Fig. 2(C), the PAI image was presented in pseudo-color and then superimposed on the gray-scale US image. The inflammation in soft tissues surrounding the joint can be seen from the optical contrast presented by PAI.

In comparison with the images from normal joints, there are increased image intensities in the joints affected by arthritis, which is believed to be mainly due to angiogenesis in the inflammatory tissues. To achieve quantitative comparison, the intensities in the ROI were averaged for each PAI image. With the PAI images from a group of 12 arthritic joints and another group of 12 normal joints, an average and a standard deviation of the image intensities for each group were computed, as shown in Fig. 3(A), where we can see clearly enhanced image intensities in the arthritic joints in comparison with the normal joints.

For clinical evaluation of the degree of arthritis, the diameter of each imaged joint was also measured at the same time when the joint was imaged. As shown in Fig. 3(B), the average size of the ankle joints from the arthritic group is much larger than that of the normal group, indicating strong synovitis as a result of adjuvant injection. To further confirm arthritis and angiogenesis in the ankle joints of the AIA rats, representative photomicrographs of an arthritic and a normal rat tarsal (ankle) joints are also given in Fig. 4. The photomicrograph from the normal control shows clear space between tarsal bones with normal, sparsely cellular, inter and periarticular fat and connective tissue (arrows). In the photomicrograph from the arthritic joint, there is inflammation, fibroplasia, and neovascularization (granulation tissue) between and around tarsal bones (arrows). In Fig. 4(C), which is the higher magnification from the area marked with asterisk in Fig. 4(B), the infiltrating granulation tissue is accompanied by numerous small diameter capillaries (arrows) surrounded by many inflammatory cells (predominantly neutrophils). 


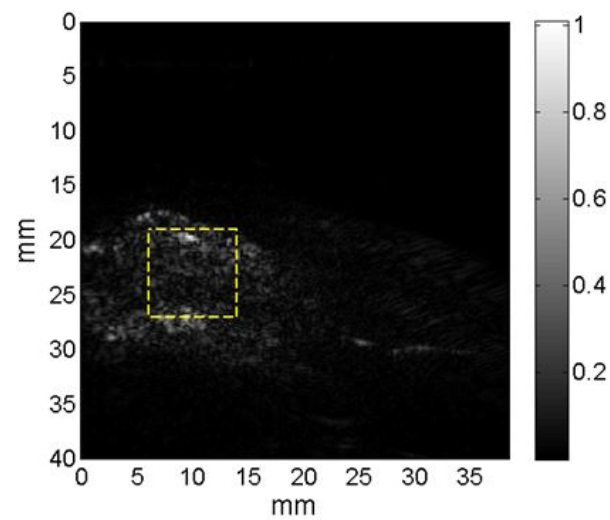

(A)

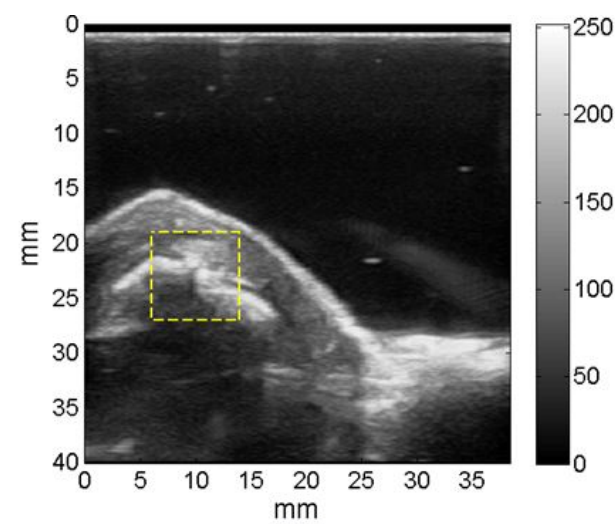

(B)

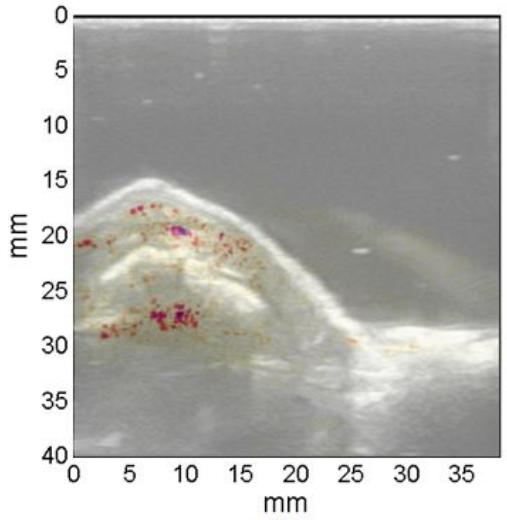

(C)

Fig. 2. PAI and US dual-modality imaging of a rat ankle joint affected by AIA. (A) Gray-scale PAI image. (B) Gray-scale US image. (C) Pseudo-color PAI image superimposed on the grayscale US image.

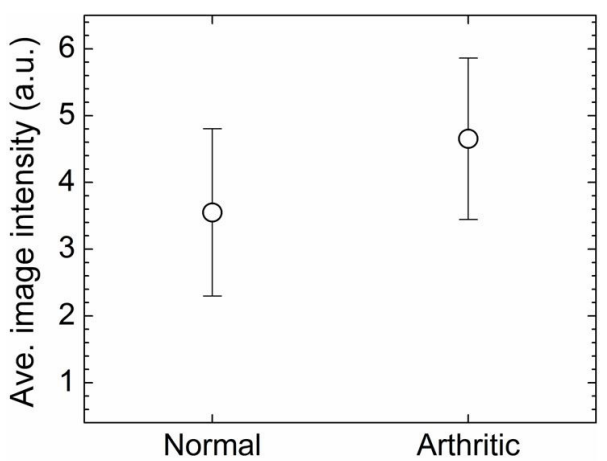

(A)

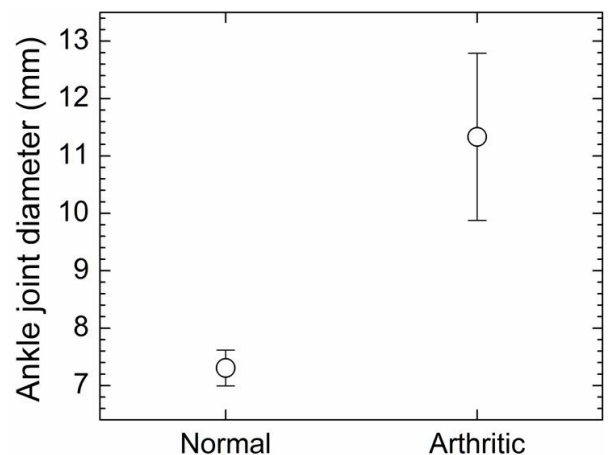

(B)

Fig. 3. (A) Average photoacoustic image intensities in the normal vs. the arthritic ankle joints. (B) Average diameters of the normal vs. the arthritic ankle joints. 


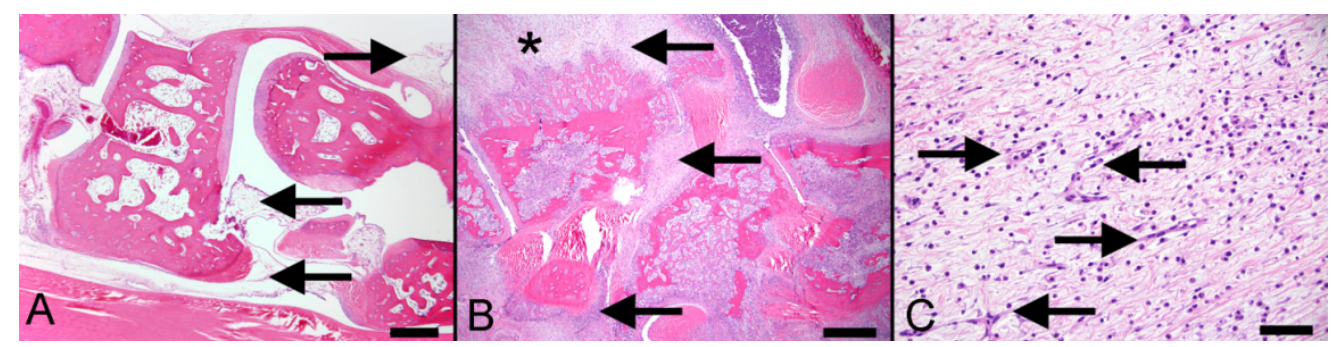

Fig. 4. Photomicrographs of a normal (A) and an arthritic (B and C) rat tarsal (ankle) joints. A and $\mathrm{B}$ : original magnification $\times 40$, bar $=500 \mu \mathrm{m}$. C: original magnification $\times 400$, bar $=$ $50 \mu \mathrm{m}$.

To demonstrate the capability of PAI in evaluating the treatment efficacy, imaging of arthritic ankle joints from the treatment group was conducted on day 35. Imaging findings were compared with the results of the arthritic joints from the non-treatment control group, as example images shown in Fig. 5. With the results from 12 joints in each group, the average and the standard deviation of PAI measurement were presented in Fig. 6(A). We can see decreased image intensities in the joints from the treatment group in comparison with the measurements from the non-treatment group, suggesting that, by evaluating the angiogenesis in arthritic tissues, the functional information presented by PAI can help monitoring the treatment efficacy. To verify the finding from PAI, the diameters of the ankle joints were measured on day 35. The averages and the standard deviations for the two groups were compared, as shown in Fig. 6(B). In comparison with the non-treatment group, the sizes of the ankle joints after being treated with the etanercept were smaller, proving less synovitis in the imaged joints as a result of the medication.

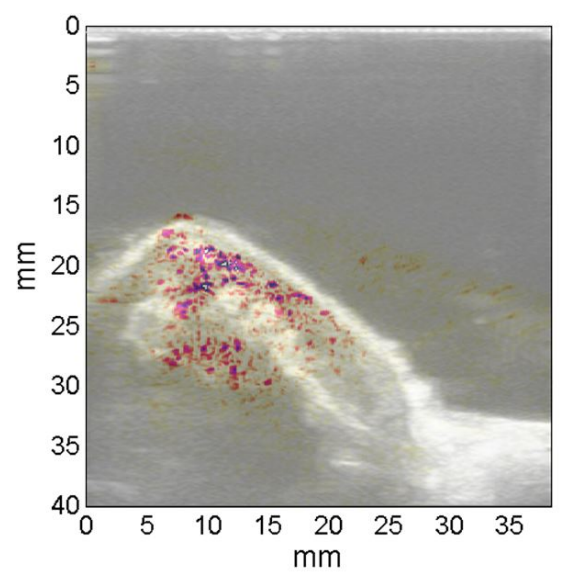

(A)

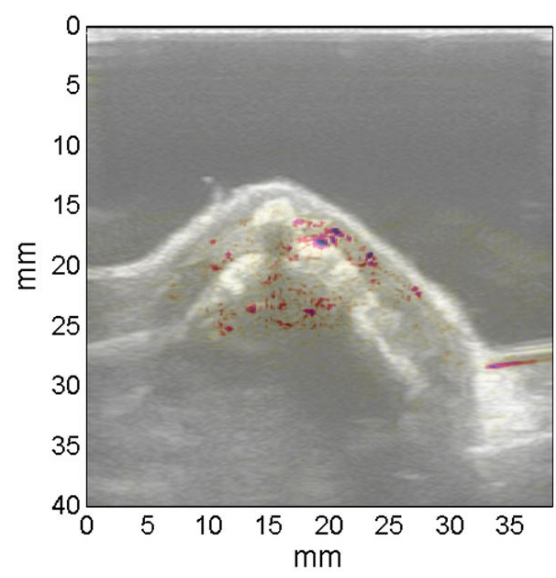

(B)

Fig. 5. Pseudo-color PA image superimposed on the gray-scale US image. (A) Image of an arthritic joint of a rat from the non-treatment control group, (B) image of an arthritic joint of a rat from the treatment group. 


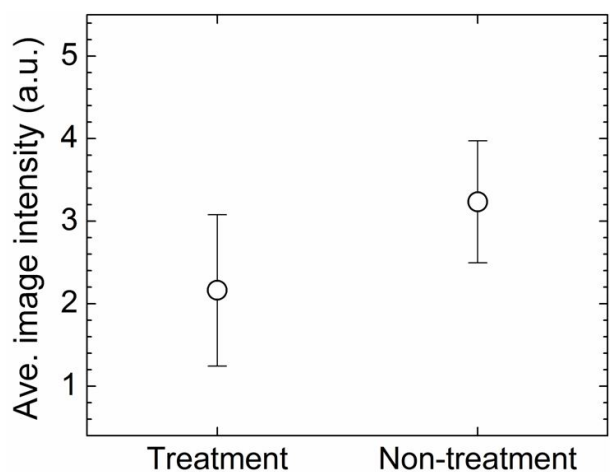

(A)

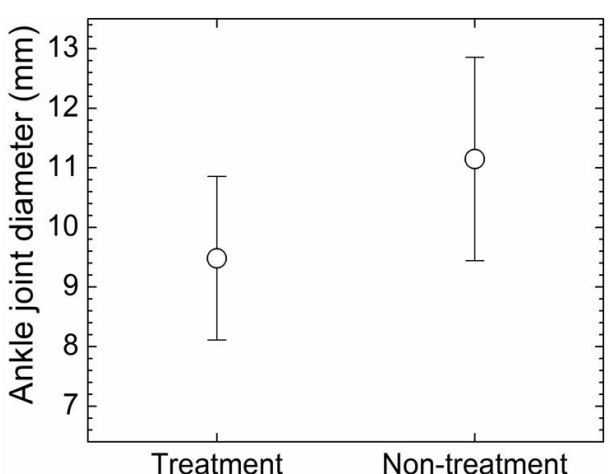

(B)

Fig. 6. (A) Average photoacoustic image intensities in the treated vs. the untreated arthritic ankle joints. (B) Average diameters of the treated vs. the untreated arthritic ankle joints.

In Fig. 7, example microPET images taken from two rats, one from the treatment group (A, B and C) and the other from the non-treatment group (D, E and F), are shown. For each rat, three images were acquired at three different time points, i.e. day 0 , day 20 and day 35, respectively. On day 0 when both rats were normal, there were no much ${ }^{11} \mathrm{C}-\mathrm{PBR} 28$ uptakes in the ankle joints. On day 20 when both rats were affected by AIA, there were significantly increased ${ }^{11}$ C-PBR28 uptakes in the arthritic ankle joints. On day 35 , we can see clearly decreased uptake of ${ }^{11} \mathrm{C}$-PBR28 in the ankle joints after the rat was treated with etanercept [Fig. 7(C)]. In comparison, the image taken on day 35 from the rat that was not treated with any medication still shows strong uptake of ${ }^{11} \mathrm{C}-\mathrm{PBR} 28$ in the ankle joints. The PET result in Fig. 7, showing the same trends as the PAI result, verified the synovitis in the ankle joints after adjuvant injection, as well as the alleviated condition after the AIA rats were treated with etanercept medication.

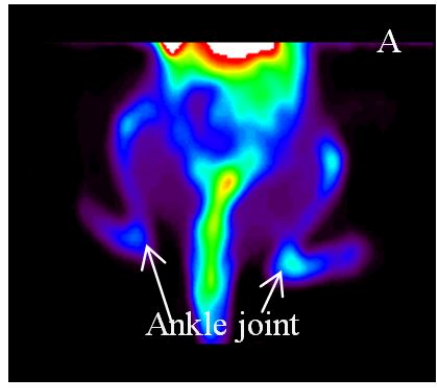

Day 0

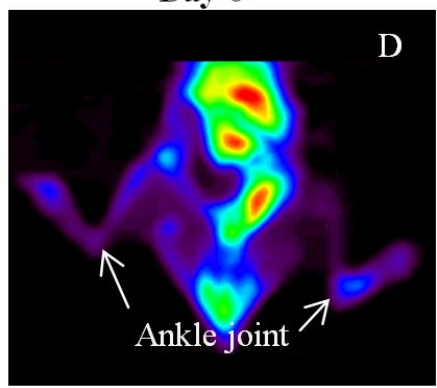

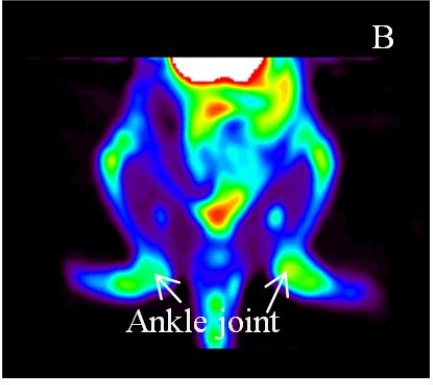

Day 20

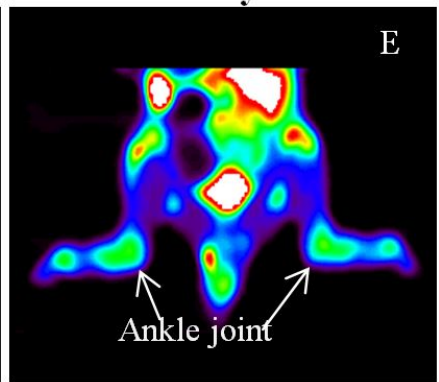

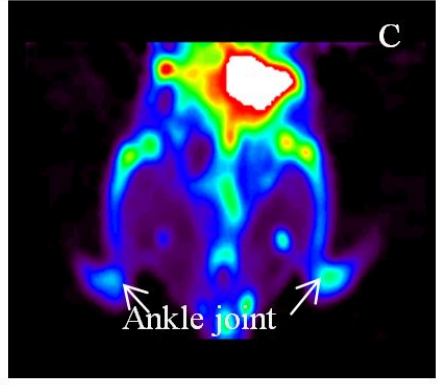

Day 35

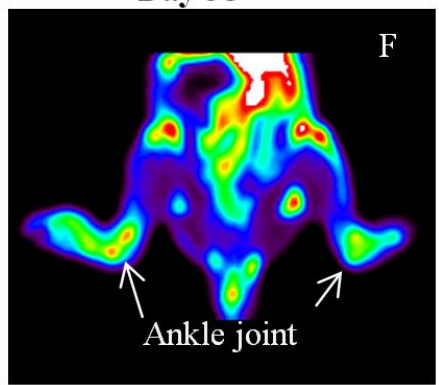

Fig. 7. MicroPET images covering the rear part of the rat body, including the ankle joints that were the targets for imaging. A, B and C are images of a rat from the treatment group taken on day 0 (normal), day 20 (arthritic) and day 35 (treated), respectively. D, E and F are images of a rat from the non-treatment group taken on day 0 (normal), day 20 (arthritic) and day 35 (untreated), respectively. 


\section{Conclusion}

Through the study on a rodent model similar clinically and pathologically to human rheumatoid arthritis, we have explored the feasibility of PAI, in combination with more established US technology, for diagnostic imaging and therapeutic monitoring of inflammatory joint diseases. Based on the optical absorption contrast that has high sensitivity in characterizing the hemodynamic properties in soft tissues, PAI showed promise in evaluating angiogenesis, as a functional biomarker of the synovitis, as well as its progression which can be a direct indicator of the treatment response. As demonstrated by the imaging experiments on rats, PAI images can differentiate the AIA joints from the normal, and can assess the functional change in the arthritic joints after the anti-rheumatic drug was given. Findings from PAI were also confirmed by histopathological examination, clinical evaluation, and microPET which, used as a gold standard, was conducted on the same animals. This study demonstrates that PAI, in conjugation with conventional US, provides opportunities to resolve the difficulties in characterization and therapy of inflammatory arthritis. Moreover, conducting functional and morphological imaging simultaneously, especially when realized with a low-cost yet powerful PAI and US dual system, may help reduce the high medical expenditures on the clinical management of arthritis, a central object of health care reform.

Statistical analysis of the data, as shown in Figs. 3 and 6, show large standard deviations. One reason is the large animal-to-animal differences in the responses to adjuvant injection and anti-rheumatic medication. This reason is very likely, as the clinical evaluation by measuring the joint diameter also shows large standard deviations. Other possible reasons include animal motion, laser output fluctuation, and the system error due to the 2D imaging. Since the functional change in an arthritic joint contains 3D volumetric information, 2D imaging through a single plane in the joint may lead to large standard deviations in quantitative measurements. Future work includes clinical evaluation on patients affected by rheumatoid arthritis. Because both PAI and US are noninvasive and nonionizing, transferring from a lab tool for animals to a clinical device for patients is promising. Considering the sizes of human peripheral joints are larger than those of the small animals, laser light at 532-nm wavelength may not penetrate sufficiently for noninvasive imaging of a human joint as a whole organ. In that case, laser light in the near-infrared spectrum region with excellent penetration in tissues should be employed.

\section{Acknowledgments}

The authors thank Alisa Koch, MD, at University of Michigan for her support in the animal model, and Ingrid L. Bergin for her contribution in histopathological examination. This study is sponsored by NIH grants R01AR055179 and R01AR060350. 